\title{
Multi-Species Continuous Gas Detection with Supercontinuum Laser at Telecommunication Wavelength
}

\author{
Adamu, Abubakar Isa; Dasa, Manoj Kumar; Bang, Ole; Markos, Christos
}

Published in:

IEEE Sensors Journal

Link to article, DOI:

10.1109/JSEN.2020.2993549

Publication date:

2020

Document Version

Peer reviewed version

Link back to DTU Orbit

Citation (APA):

Adamu, A. I., Dasa, M. K., Bang, O., \& Markos, C. (2020). Multi-Species Continuous Gas Detection with Supercontinuum Laser at Telecommunication Wavelength. IEEE Sensors Journal, 20(18), 10591 - 10597. https://doi.org/10.1109/JSEN.2020.2993549

\section{General rights}

Copyright and moral rights for the publications made accessible in the public portal are retained by the authors and/or other copyright owners and it is a condition of accessing publications that users recognise and abide by the legal requirements associated with these rights.

- Users may download and print one copy of any publication from the public portal for the purpose of private study or research.

- You may not further distribute the material or use it for any profit-making activity or commercial gain

- You may freely distribute the URL identifying the publication in the public portal 


\title{
Multi-Species Continuous Gas Detection with Supercontinuum Laser at Telecommunication Wavelength
}

\author{
Abubakar I. Adamu, Manoj K. Dasa, Ole Bang and Christos Markos
}

\begin{abstract}
Supercontinuum (SC) lasers provide an excellent opportunity for absorption spectroscopy by virtue of their high brightness and large bandwidth. Here we present the detection of multiple industrial toxic gasses from $1480 \mathrm{~nm}$ to $1700 \mathrm{~nm}$, with a simple custom SC laser source. Using readily available optical components, we demonstrate an all-fiber system for the detection of ammonia $\left(\mathrm{NH}_{3}\right)$ and methane $\left(\mathrm{CH}_{4}\right)$ in a $2.4 \mathrm{~m}$ long hollow-core photonic bandgap fiber with 20 - $\mu \mathrm{m}$ core diameter. The responsivity, selectivity, and performance of the system for continuous detection are demonstrated.
\end{abstract}

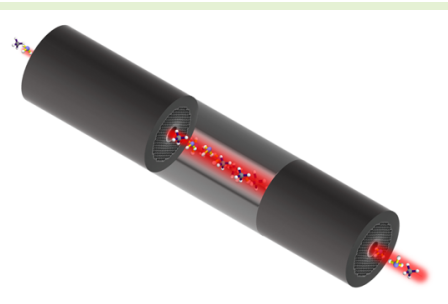

Index Terms-Optoelectronic and photonic sensors, Gas sensors, Environmental monitoring, Optical fiber sensors.

\section{INTRODUCTION}

$\mathrm{S}$ UPERCONTUNUUM (SC) lasers are spatially coherent so-called white light lasers with an enormous bandwidth covering several octaves and an extreme spectral brightness, which is orders of magnitude higher than even synchrotrons [1]. The unique properties of the SC laser has allowed it to make a remarkable impact in the fields of applied spectroscopy [2], food control [3], biomedical sensing applications [4], [5], photo-acoustic and optical coherence tomography imaging [6]-[9], telecommunication [10], inspection of optical components and semiconductor chips [11]-[13], to name a few. The majority of the laser sources used in the above-mentioned reports are fiber-based, which makes them compact and robust and therefore ideal for also operation in harsh environments, such as in stand-off detections of explosives and chemicals [14], [15].

Gas sensors based on optical methods are remarkable for their longevity, high sensitivity, selectivity, and immunity to catalyst poisoning and environmental changes [16]. Additionally, their very short response time enables on-line real-time gas detection. One of the optical sensing techniques is spectroscopy, such analysis primarily involves techniques

This work is supported by Innovation Fund Denmark (6150-00030A \& 8090-00060A), Det Frie Forskningsråd (DFF) (8022-00091B) and European Union's Horizon 2020 research and innovation programme under the Marie Sklodowska-Curie grant (agreement No 722380)

A. I. Adamu, M. K. Dasa, O. Bang and C. Markos are with the Department of Photonics Engineering, Technical University of Denmark, DK- 2800, Lyngby Denmark.

$\mathrm{O}$. Bang and C. Markos are with NORBLIS IVS, Virumgade 35D, DK-2830 Virum, Denmark.

O. Bang is with NKT Photonics, Blokken 84, Birkerød 3460, Denmark

A. I. Adamu is the corresponding author, email abisa@fotonik.dtu.dk which rely on absorption and emission spectrometry [16]. Absorption spectroscopy relies on the Beer-Lambert law, which provides the relation for concentration-dependent molar absorptivity of photons at specific wavelengths [17], which is distinct for every gas because of their absorption fingerprints, thus enabling for high selectivity. This selectivity, coupled with the broadband spectrum of the SC source, allows for multi-species gas detection [18], [19]. Comparatively, absorption-based optical gas sensors have advantage over electrochemical based gas sensors or metal oxide-based sensors [20], [21] which typically target only a specific gas. Most cost-effective and commonly used gas sensors today are metal oxide sensors [16], whose device response depends on the redox reaction between the target gas and the oxide surface [16], [22]. This practically means that they suffer from a lack of selectivity [23] and short lifetime as a result of deteriorating metal oxide surface. Although such sensors can be very sensitive, their high sensitivity comes at a cost, because they often require high operation temperatures of up to typically a few hundred degrees Celsius [24], [25]. Furthermore, there is a constraint on other $\mathrm{NH}_{3}$ sensors as to the maximum detection limit, with photoionization detectors having a response to 1000 ppm [26] and electrochemical based $\mathrm{NH}_{3}$ sensors saturating at only $100 \mathrm{ppm}[26],[27]$.

Unlike the aforementioned chemical reaction-based technique, spectroscopy-based sensors target the specific absorption of molecules, and because every gas has its distinct and unique absorption spectrum, the sensor can be highly selective. Thus, rather than cross-examining the response from arrays of sensors, one can deploy a single broadband SC source and target multiple gases. Other results have demonstrated the use of hollow core fiber for gas sensing, such as photothermal gas sensors [28] and very recently, a 
study from the same group on mode-phase-difference based photothermal sensing [29] demonstrated acetylene detection with non-commercial fibers. Although remarkable performance has been reported in the literature, there is no report on multiple continuous gas detection with single laser source. Our cheap and easy-to-build SC source provides a suitable solution for such applications since tunable quantum cascade lasers are not available in the near- IR.

Here we demonstrate a home-built SC laser suitable for the simultaneous detection of ammonia $\left(\mathrm{NH}_{3}\right)$ and methane $\left(\mathrm{CH}_{4}\right)$. These two gasses are chosen because they are the most predominant gasses emitted in places of intense agriculture, such as dairy barns [30], livestock farming [31] and fertilizer industry [23]. Exposure to $\mathrm{NH}_{3}$ can lead to many medical complications ranging from severe nasal irritation to death, depending on the concentration and the exposure time [23]. Farming being the major contributor of $\mathrm{NH}_{3}$ in the atmosphere accounts for over $50 \%$ of global $\mathrm{NH}_{3}$ emission [31], [32]. Furthermore, about $50 \%$ of this $\mathrm{NH}_{3}$ emission ends up as a form of environmental pollutant. Agriculture is also the second highest contributor to $\mathrm{CH}_{4}$ emission, accounting for up to $31 \%$ of total emission in the US for example [33]. The emission of $\mathrm{CH}_{4}$ is considered among the primary contributors to Earth warming [34]. Therefore, having a gas sensor that can continuously detect and monitor the emissions of these gases is of great importance. We present a cost-effective method for detecting these two gases using optical-based absorption spectroscopy in a hollow-core photonic band gap (HCPBG) fiber. For any optical fiber sensor to be practical and deployable in the field, it is necessary for it to be compact and all-fiberized. To this end, we demonstrate a complete all-fiber system based on commercial telecom range components, where gas entry and exit into the $20 \mu \mathrm{m}$ hollow core is achieved with a mechanical splice, between the single-mode fiber (SMF) (SMF-28, Corning) and HCPBG. One advantage of operating in the telecommunication wavelength regime is the availability of high-quality components at low-cost, thanks to the advanced level of development in optical telecommunication technology. Therefore, the overall developed system costs significantly less than its commercial counterpart. In this work, we fully characterize the proposed sensing system and we demonstrate its performance for continuous gas monitoring and analyse different parameters such as response time, SC tunability, repeatability, selectivity, and sensitivity. The proposed system also can detect very wide-range of concentrations- showing linear response to concentrations as high as $100 \%$ for $\mathrm{CH}_{4}$ and $80 \%\left(8 \times 10^{5}\right.$ ppm) for $\mathrm{NH}_{3}$.

\section{SC LASER FOR Ammonia ANd Methane Detection}

The excitation laser used for the experiment is a relatively easy-to-build fiber-coupled, in-house made SC laser. The SC laser uses a directly modulated telecom-range diode laserbased amplifier (MLT-PLR-OEM20, Manlight) operating at $\sim 1550 \mathrm{~nm}$ as a pump laser with $3.2 \mathrm{~ns}$ pulse length duration. The pump laser is driven by an external pulse generator (TG2000, AIM-TTi), which is tunable from $30 \mathrm{kHz}$ to 100
$\mathrm{kHz}$. This tunability is vital for our application because the peak power is consequential in determining the broadening of the SC spectrum. The pulses from the laser are used to pump a standard dispersion-shifted fiber (DSF) (DCF4, Thorlabs) with a ZDW close to $1550 \mathrm{~nm}$; this means our pump is very close to the ZDW, which is essential for standard SC generation [35]. The fiber output of the pump laser is directly spliced using a commercial splicer (FSM-100P+, Fujikura) on to the DSF so as to maximize the coupling efficiency and also for the ease of handling. In order to avoid back reflections from the end facet of the output fiber, the output fiber was angle cleaved - setting a cleaving angle of about 8 degrees in the tension cleaver (CT-100, Fujikura).

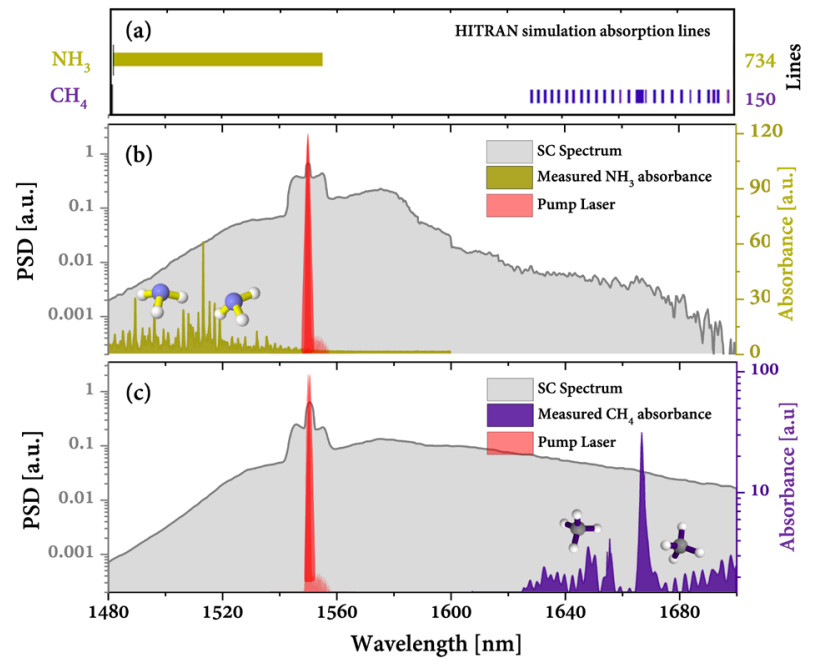

Fig. 1. (a) Simulation of molecular absorption lines of $\mathrm{NH}_{3}$ and $\mathrm{CH}_{4}$ from the Hitran2016 database [36]; Number of discrete spectral lines shown on right. (b) $\mathrm{SC}$ generated for $\mathrm{NH}_{3}$ sensing, overlaid with measured $\mathrm{NH}_{3}$ absorbance (c) SC spectrum for $\mathrm{CH}_{4}$ sensing, overlaid with measured absorbance of $\mathrm{CH}_{4}$.

The angle-cleaved end of the fiber is placed in the ferrule inside the gas-cell shown in Figure 2. Details of the experimental setup are explained in the next section III.

The output spectrum of the SC is tailored to match the absorption bands of the species by switching between two settings. For $\mathrm{NH}_{3}$ detection, we use a repetition rate of $30 \mathrm{kHz}$ and a seed current of $1700 \mathrm{~mA}$ to increase the power spectral density (PSD) below the pump - since the region of interest (as seen in Figures 1(a) and 1(b)) is from about $1480 \mathrm{~nm}$ to 1550 $\mathrm{nm}$. For $\mathrm{CH}_{4}$ detection, we use a repetition rate of $91 \mathrm{kHz}$ and a seed current of $3200 \mathrm{~mA}$, to increase the PSD above $1610 \mathrm{~nm}$ - since this is the region of interest for $\mathrm{CH}_{4}$ (as seen in Figures 1(a) and 1(c)).

\section{EXPERIMENTAL SET-UP FOR AlL-FIBER SYSTEM FOR GAS SENSING}

The applicability of SC laser described in the previous section is demonstrated for multi-species gas sensing. The angle-cleaved output fiber was placed inside a ceramic ferrule, such that the tip of the fiber is barely extruding out of the ferrules' borehole. Then the other end of the ferrule is glued with an epoxy glue. Another ferrule of same dimensions is used for the HCPBG fiber, here, the facet of the fiber is flat- 
cleaved because there is no issue of back reflection since the core is hollow (air). The HCPBG fiber is fixed into the ferrule similarly with epoxy; the two connectorized fibers are then mated with a mating-sleeve connector (Thorlabs, ADAF1). The mechanical splice is adjusted carefully to obtain high coupling efficiency between the two fibers while still maintaining some gap between the two ends to facilitate gas diffusion into the HCPBG fiber. This procedure is done carefully under optical microscope for higher precision. We found that a gap of $\sim 80 \mu \mathrm{m}$ between the SMF and the HCPBG fiber gave a coupling efficiency of $\sim 65 \%$ while maintaining sufficient path for gas diffusion. The procedure is done again at the other end of the HCPBG fiber, where an optical patch cable is mechanically spliced to the HCPBG and then connected to either an Optical spectrum analyzer (OSA) (Assetrelay, ANDO AQ6317B) or a power meter (Thorlabs, S407C) for spectral characterization and power monitoring, respectively.

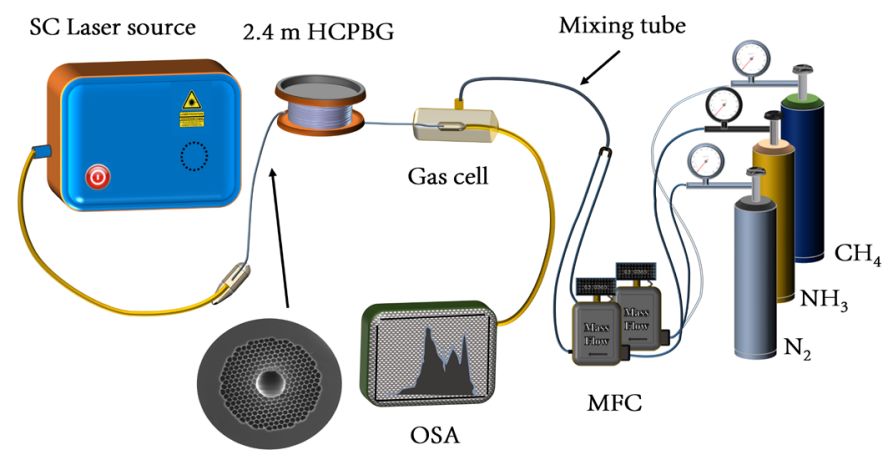

Fig. 2. Schematic of multi-species gas sensing set up: The SMF from the laser is mechanically spliced to an HCPBG fiber, the other end of the HCPBG is also mechanically spliced, and placed in a sealed gas-cell. Two MFCs are used to control the flow from $\mathrm{N}_{2}$ and either $\mathrm{CH}_{4}$ or $\mathrm{NH}_{3}$. A Scanning Electron Microscopy (SEM) image of the HCPBG fiber is shown.

One junction of the two mechanical splices is placed in a simple custom-made gas-cell; this is aimed to provide a pressure gradient in the system, so gas flows in a specific direction in the system. Gas is passed into the $2.4 \mathrm{~m} \mathrm{HCPBG}$ through the gas-cell.

Nitrogen gas $\left(\mathrm{N}_{2}\right), \mathrm{NH}_{3}$ and $\mathrm{CH}_{4}$ cylinders (from Air liquide A/S), with high purity $(99.999 \%)$ are connected to a mass flow controller (MFC) (EL-Flow, Bronkhorst). The controllers regulate the flow of gasses, enabling for the dilution of gas mixtures at various concentrations with high precision. $\mathrm{N}_{2}$ is here used both as the diluent and as background gas during the experiment. The choice of $\mathrm{N}_{2}$ is not only because $\mathrm{N}_{2}$ is the most abundant gas in open atmosphere (78.1\%), but also it has no absorption lines in our spectral region of interest. The MFC is operated at a flow rate between 5-100 standard cubic centimeters per minute $(\mathrm{sccm})$, which is within the linear operation regime of the MFC devices. It is important to mention that a sufficient length of tube, mixing tube, is used after the connecting junction of the two MFCs to ensure homogeneity of the gas mixture before it is passed into the gas-cell.

\section{Multi-gas Detection}

\section{A. Spectral Analysis}

The output spectrum of the fiber is measured using an ANDO OSA with a resolution of $0.1 \mathrm{~nm}$. Firstly, the entire chamber was purged with $\mathrm{N}_{2}$ gas, and the background spectrum is recorded, shown in red in Figs. $3 \mathrm{~b}$ and $4 \mathrm{~b}$. Then either $\mathrm{NH}_{3}$ or $\mathrm{CH}_{4}$ is passed through the chamber. The laser parameters were tuned to increase the power in the respective spectral regions as detailed in Section II and the spectrum was recorded. In the case of $\mathrm{NH}_{3}$, the spectral measurement was done at several concentrations and in all cases the spectral aborption dips are clearly visible in the $1500-1550 \mathrm{~nm}$ band, as seen in Fig. $3 \mathrm{~b}$. We calculated the absorbance, $\log \left(\mathrm{I}_{0} / \mathrm{I}\right)$, of the gases; where $\mathrm{I}_{0}$ and $\mathrm{I}$ is the transmission with pure $\mathrm{N}_{2}$ and target gas respectively. This is depicted in Fig. 3a, where the measured absorbance perfectly captures the absorption band from Hitran (with intensity cut-off of $1 \times 10^{-28} \mathrm{~cm} / \mathrm{mol}$ ). In particular, it accurately captures the strongest peak at $1531 \mathrm{~nm}$ which has been reported in many studies of ammonia near-IR spectroscopy [37], [38].
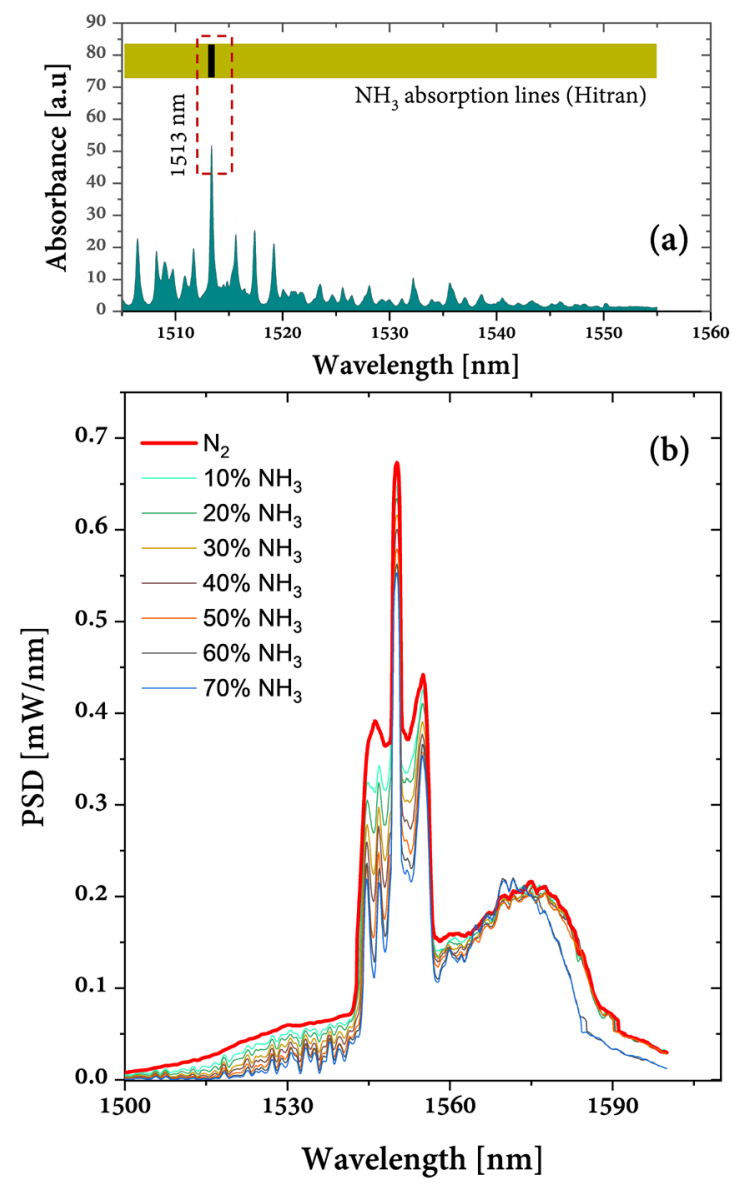

Fig. 3. (a) Absorbance of $\mathrm{NH}_{3}$ ploted along with Hitran simulation using $1 \times 10^{-}$ ${ }^{28} \mathrm{~cm} / \mathrm{mol}$ intensity cut-off, (b) Spectrum of $\mathrm{NH}_{3}$ at various concentrations. Red curve is the spectrum of the background $\left(\mathrm{N}_{2}\right)$. The system is flushed with $\mathrm{N}_{2}$ after every measurement. Percentage of $\mathrm{NH}_{3}$ for each measurement is shown in the legend.

As for $\mathrm{CH}_{4}$, the spectrum shown in Fig. 4 has absorption dips in the wavelength region from $1620 \mathrm{~nm}$ to $1700 \mathrm{~nm}$, which matches the absorbtion band in the HITRAN simulations of Fig. 1(a). Here also, the measured absorbance captures the 
strongest peak at $1666 \mathrm{~nm}$ as shown in Fig 4a. For both $\mathrm{NH}_{3}$ and $\mathrm{CH}_{4}$ however, each and every individual absorption line cannot be fully resolved because the actual absorption lines are much narrower than the maximum resolution of $0.1 \mathrm{~nm}$ of the OSA used. Therefore, in order to quantify the relative change in transmitted light between $\mathrm{N}_{2}$ and any other gas mixture, one could calculate the area under the curve (spectrum) and compute the differences. In other words, we can alternatively monitor the changes in output power using a power meter. This will also permit for real-time continuous measurement [39].
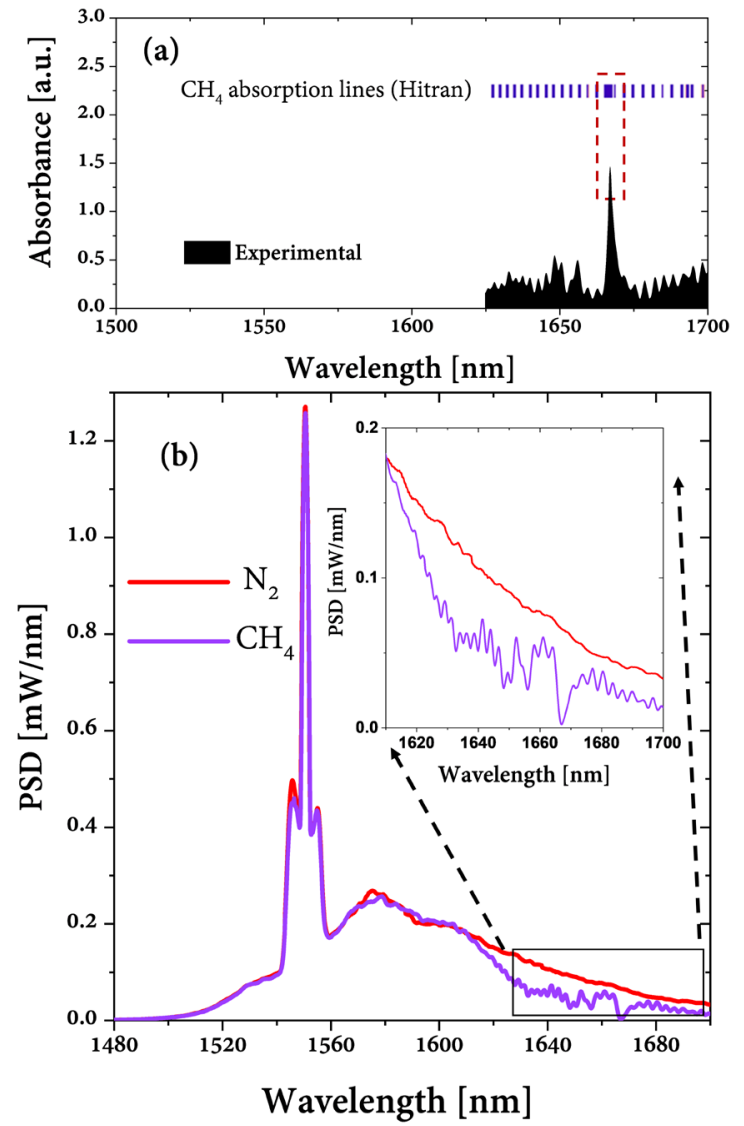

Fig. 4. (a) Hitran simulation of $\mathrm{CH}_{4}$ with line intensity cut-off of $1 \times 10^{-28}$ $\mathrm{cm} / \mathrm{mol}$ and measured absorbance of $\mathrm{CH}_{4}$, with peak at $1666 \mathrm{~nm}$ accurately captured. (b) The spectrum after the all-fiber gas system; Red spectrum shows the spectrum after $100 \% \mathrm{~N}_{2}$ is passed. Purple spectrum is measured after $50 \%$ $\mathrm{CH}_{4}$, and $50 \% \mathrm{~N}_{2}$ diluted gas is flushed into the fiber. Inset shows a zoom of the spectrum from $1610 \mathrm{~nm}$ to $1700 \mathrm{~nm}$.

\section{B. Power Analysis}

To have a continuous measurement of the gas in the system, a thermopile IR power meter (Thorlabs, S470C) was deployed, and the power is monitored in real-time. The power remained constant when pure $\mathrm{N}_{2}$ is continuously flushed through the system. As seen in Fig. 5, after each $\mathrm{NH}_{3}$ mixture, pure $\mathrm{N}_{2}$ is passed into the system, and the output power always recovers to the initial position, showing the reliability of the system. When a $5 \% \mathrm{NH}_{3}$ mixture was injected, the power dropped and saturated at a specific value. When a higher concentration of $\mathrm{NH}_{3}$ was used, the power drops to an even lower level. That is to say, we monitored the changes in the transmitted light (output power) at various concentrations of $\mathrm{NH}_{3}$. The inset in Fig. 5 shows a linear fit to the power response, calculated as the percentage reduction in transmitted power, with the shaded region showing a 95\% confidence band.
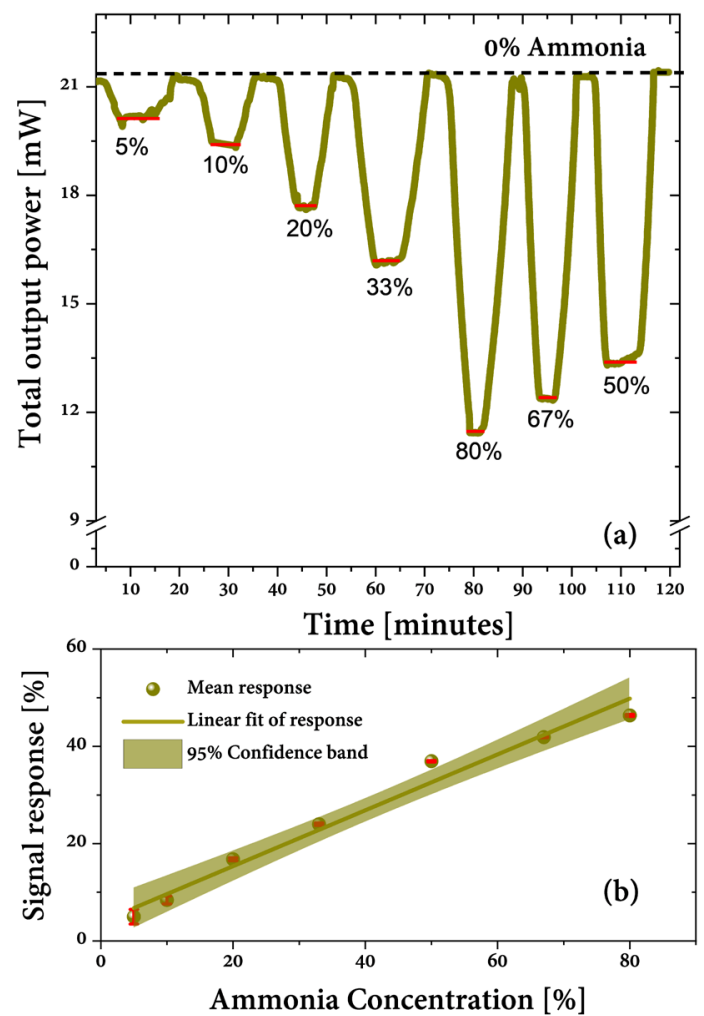

Fig. 5. (a) Continuous power log of $\mathrm{NH}_{3}$ gas sensing: diluted concentrations from $5 \%$ to $80 \%$. $\mathrm{N}_{2}$ gas is passed into the system after each $\mathrm{NH}_{3}$ mixture is flushed through the fiber. At $0 \% \mathrm{NH}_{3}$, only $\mathrm{N}_{2}$ is in the fiber core. Red bars show the saturated power level for various concentrations and span in time, used in calculating signal response in (b). (b) Shows signal response of $\mathrm{NH}_{3}$ at different concentrations, with the shaded area showing a $95 \%$ confidence band. Error bars in red.

A similar procedure was followed for $\mathrm{CH}_{4} . \mathrm{N}_{2}$ is then mixed with $\mathrm{CH}_{4}$ from $0 \%$ to $100 \%$. The continuous power log is shown in Fig. 6, and the relative decay in power (signal response) is plotted with the purple shaded area showing the 95\% confidence band in Fig. 6(b). Similar to Fig. 5(b), the response is defined as the relative decay in power from the level for pure $\mathrm{N}_{2}$ (in this case $9.2 \mathrm{~mW}$ ), the saturated region for each concentration is shaded in red. 

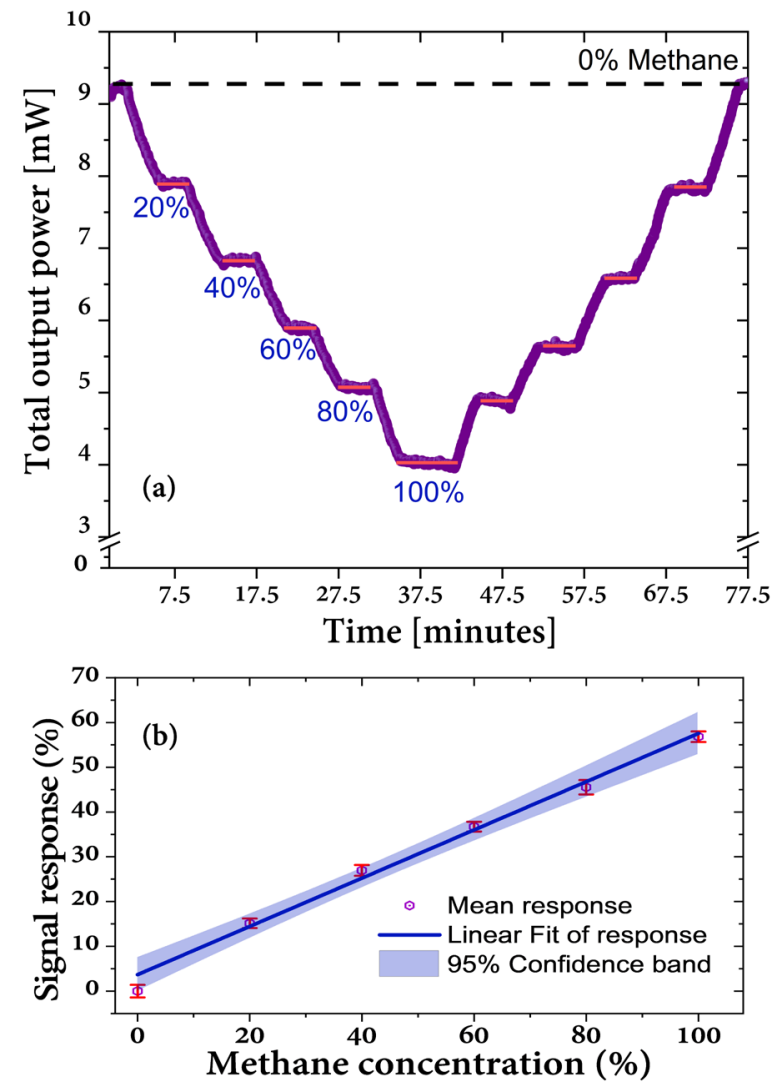

Fig. 6. (a) Continuous power log of $\mathrm{CH}_{4}$ gas sensing: $\mathrm{CH}_{4}$ mixture of concentrations from $0-100 \%$, measurement is continuous with only the MFC flows changed. The power remains constant once the mixture has filled the HCPBG. Red bars show the saturated regions at various concentrations, which is used in calculating signal response for methane in (b). The bars signify the saturated power level and the span used in calculation the signal response in (b) (b) Shows the best linear fit of the gas response vs. concentration, with the purple shaded region showing the $95 \%$ confidence band.

\section{Response time, RepeatABiLITY AND DETECTION LIMITS}

\section{A. Response time}

In a continuous gas monitoring system, a sensor ought to be able to detect the concentration of the target gas at any given instant. In the all-fiber system, the response measured is always of the gas inside the $2.4 \mathrm{~m}$ long hollow-core of the fiber at any given instant. However, in this particular demonstration, although the device gives the measurement at every instant, the target gas from the gas cylinder takes time to fill the tubes and dilute with the background gas, and it also takes time to fill the fiber core. Thus, the gas dynamics is dependent on the flow rate of the MFCs, the fiber length, and also the length of our mixing tube, which is $\sim 1.5 \mathrm{~m}$. In diluting the gases, the nominal flow changes from one concentration to another; for example, to dilute $\mathrm{NH}_{3}$ by $50 \%$, the MFC was set to give out $50 \mathrm{sccm}$ of $\mathrm{N}_{2}$ per minute, and the second MFC is also set to $50 \mathrm{sccm}$. This gives a total flow of $100 \mathrm{sccm}$ after the dilution. In case of $80 \% \mathrm{NH}_{3}$ however, the $\mathrm{NH}_{3} \mathrm{MFC}$ was set to $50 \mathrm{sccm}$ while the $\mathrm{N}_{2} \mathrm{MFC}$ was set to $13 \mathrm{sccm}$, giving a total mass flow of $63 \mathrm{sccm}$, this gives a longer response time of about 5.72 minutes compared to when the total mass flow was $100 \mathrm{sccm}$. To show this, a section of the plot in Fig. 5 is analyzed in Fig. 7 (a). The fact that the response time is dependent on the flow of gas, but not its concentration, is clearly seen by comparing the cases (i) and (ii) in Fig. 7, which both have the same flow rate $(100 \mathrm{ccc} / \mathrm{m})$ and response time (3.4 minutes), but different concentrations of $\mathrm{NH}_{3}$. It is noteworthy that this long duration of gas mixing before diffusing into the fiber can easily be improved in a real-world system, where the target gas is delivered directly into the gas cell (with a vent-fan for example). Such that, only the time to fill a given length of the fiber is important, which is often a trade-off between the absorbance and response time. A previous study has shown that a response time as low as 3 seconds is attainable [40], albeit, this requires sophisticated femtosecond micromachining along the length of the fiber for gas diffusion.
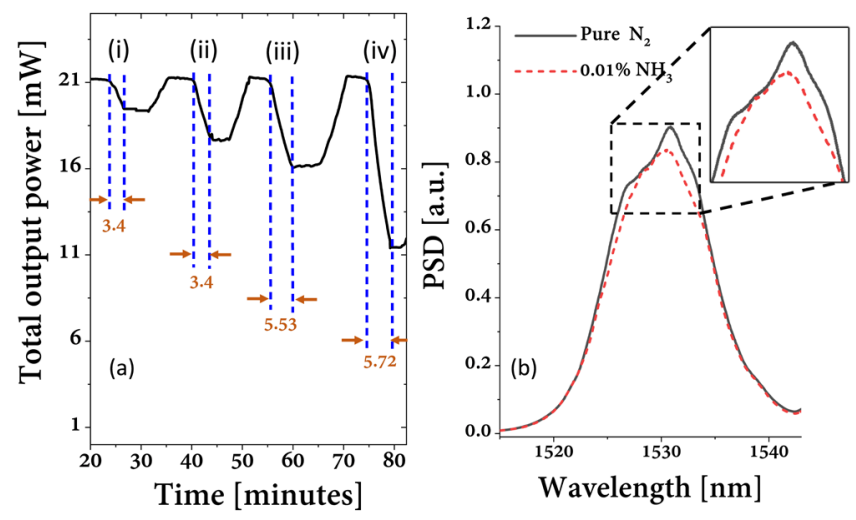

Fig. 7. (a) Shows the time it takes for the power to saturate from a section of the plot in Fig. 5. Duration for power to the plateau is taken as response time. Total flow rates of case (i) and (i) is $100 \mathrm{sccm}$, (iii) is $75 \mathrm{sccm}$ and (iv) is 63 sccm. (b) Spectra after a band-pass filter with $1530 \mathrm{~nm}, 10 \mathrm{~nm}$ FWHM is used. Spectrum in black is $\mathrm{N}_{2}$ and dotted red shows the $0.01 \% \mathrm{NH}_{3}$ spectrum.

\section{B. Noise and Detection limit}

Although SC lasers are known for their pulse to pulse intensity fluctuations [41], the effect of the pulse to pulse fluctuations is significantly reduced when data is taken from the average of many pulses [2]. In this paper, the response from both the OSA and power meter sensor is averaged over $\sim 15,000$ pulses, which significantly increases the signal to noise ratio. With an OSA integration time of 1 second, the $\mathrm{NH}_{3}$ and $\mathrm{CH}_{4}$ spectra will be an average over a total of 30,000 and 91,000 pulses, respectively, due to the different repetition rates used in the two settings.

We compute the standard deviation (STD) of power log from the laser (after the mechanical splices) for 4 minutes- duration for half-cycle measurement (figure 8(a)), The STD for shortterm stability is $1.57 \times 10^{-5}$. Which is similar to our long term stability for 6 hours. The Allan-werle deviation of the system for 6 hours stability measurement shows a theoretical detectable limit of 4 ppm as shown in Fig. 8(c). Other sources of noise, such as white noise from the detector, room temperature fluctuations, etc. were not considered.

A gas mixture of $\mathrm{N}_{2}$ and $0.01 \% \mathrm{NH}_{3}$ (Air Liquide $\mathrm{A} / \mathrm{S}$ ) was detected using the proposed system. The power meter reading did not show a noticeable difference when the entire spectrum is recorded, however, when a $1530 \mathrm{~nm}$ band-pass filter was 
deployed at the output, a distinct difference in amplitude could be observed between the background and 0.01\% NH3 (Fig. 7(b)) signal. Thus, the addition of filters could further improve the sensitivity, but not without compromising the all-fiberized system. Therefore the gas detection and monitoring was performed without any bulk components such as optical filters.
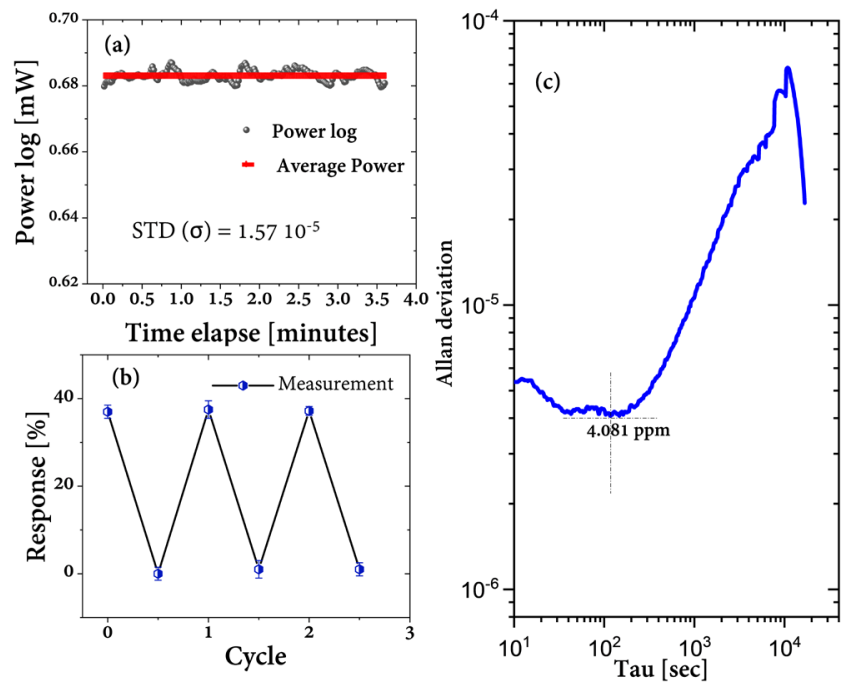

Fig. 8. (a) Shows the stability of laser over 4 minutes. (b) Measurement by repeatedly passing of $50 \% \mathrm{NH}_{3}$ and recovery after passing $\mathrm{N}_{2}$. The demonstration of 2 and a half cycles shown in figure 8(b) was carried with a $50 \% \mathrm{NH}_{3}$ mixture. (c) The Allan-Werle plot for ammonia sensing, showing the calculated detection limit, with optimal averaging time of $100 \mathrm{~s}$.

\section{Repeatability}

For any sensor, reliability is imperative; i.e., it must give consistent results when being used repeatedly. The proposed system is highly repeatable. We demonstrate this by taking a cycle of measurements for a fixed concentration and $\mathrm{N}_{2}$. The signal response of the gas always returns to a fixed value at given. Unlike metal oxides, there is no saturation of catalytic residue. Figure 8 (b) shows a response measurement for 2 and a half cycles of the system while passing $50 \%$ concentration of $\mathrm{NH}_{3}$ mixture followed by $\mathrm{N}_{2}$. It can be seen that the sensor shows consistent characteristics, thereby showcasing its repeatability.

\section{CONCLUSION}

A compact and all-fiber gas sensor for multi-species gas detection based on readily available and off-the-shelf optical components is proposed. The all-fiber gas sensor is based on a simple home-built SC laser, which is cheap compared to commercially available SC sources. By tuning the peak power of the pump laser, the output spectrum of the SC is tailored to target the absorption features of two different gases within the telecommunication regime.

The results demonstrated here shows the possibility of targeting gasses such as $\mathrm{NH}_{3}$ and $\mathrm{CH}_{4}$, which are of great importance in the agricultural and farming sector. The proposed system is highly reliable for continuous monitoring, selective, cheap and sensitive to concentrations as low as 4 ppm when estimated using Allan-werle analysis. Effort can be given to further miniaturize the system by devising simple exhaust points on the gas-cell to pass ambient air for realworld applications; such will further improve the response time to less than a minute.

\section{ACKNOWLEDGMENT}

The authors would like to thank Kyei Kwarkye, Mikkel Jensen, Getinet Woyessa \& Christian R. Petersen for fruitful discussions

\section{REFERENCES}

[1] C. R. Petersen, P. M. Moselund, L. Huot, L. Hooper, and O. Bang, "Towards a table-top synchrotron based on supercontinuum generation," Infrared Physics \& Technology, vol. 91, pp. 182-186, Jun. 2018, doi: 10.1016/j.infrared.2018.04.008.

[2] C. F. Kaminski, R. S. Watt, A. D. Elder, J. H. Frank, and J. Hult, "Supercontinuum radiation for applications in chemical sensing and microscopy," Appl. Phys. B, vol. 92 , no. 3 , p. 367 , Aug. 2008 , doi: $10.1007 /$ s00340008-3132-1.

[3] K. Eslami Jahromi et al., "A Broadband Mid-Infrared Trace Gas Sensor Using Supercontinuum Light Source: Applications for Real-Time Quality Control for Fruit Storage," Sensors, vol. 19, no. 10, p. 2334, Jan. 2019, doi: $10.3390 / \mathrm{s} 19102334$.

[4] A. Labruyère, A. Tonello, V. Couderc, G. Huss, and P. Leproux, "Compact supercontinuum sources and their biomedical applications," Optical Fiber Technology, vol. 18, no. 5, pp. 375-378, Sep. 2012, doi: 10.1016/j.yofte.2012.08.003.

[5] M. K. Dasa, C. Markos, J. Janting, and O. Bang, "Multispectral photoacoustic sensing for accurate glucose monitoring using a supercontinuum laser," $J$. Opt. Soc. Am. B, JOSAB, vol. 36, no. 2, pp. A61-A65, Feb. 2019, doi: 10.1364/JOSAB.36.000A61.

[6] N. M. Israelsen et al., "The value of ultrahigh resolution OCT in dermatology - delineating the dermo-epidermal junction, capillaries in the dermal papillae and vellus hairs," Biomed. Opt. Express, BOE, vol. 9, no. 5, pp. 2240-2265, May 2018, doi: 10.1364/BOE.9.002240.

[7] I. Hartl et al., "Ultrahigh-resolution optical coherence tomography using continuum generation in an air-silica microstructure optical fiber," Opt. Lett., OL, vol. 26, no. 9, pp. 608-610, May 2001, doi: 10.1364/OL.26.000608.

[8] Y. N. Billeh, M. Liu, and T. Buma, "Spectroscopic photoacoustic microscopy using a photonic crystal fiber supercontinuum source," Opt. Express, $O E$, vol. 18, no. 18, pp. 18519-18524, Aug. 2010, doi: 10.1364/OE.18.018519.

[9] M. K. Dasa et al., "All-fibre supercontinuum laser for in vivo multispectral photoacoustic microscopy of lipids in the extended near-infrared region," Photoacoustics, vol. 18, p. 100163, Jun. 2020, doi: 10.1016/j.pacs.2020.100163.

[10] K. Mori, K. Sato, H. Takara, and T. Ohara, "Supercontinuum lightwave source generating $50 \mathrm{GHz}$ 
spaced optical ITU grid seamlessly over S-, C- and Lbands," Electronics Letters, vol. 39, no. 6, pp. 544-546, Mar. 2003, doi: 10.1049/el:20030344.

[11] M. Lehtonen, G. Genty, and H. Ludvigsen, "Absorption and transmission spectral measurement of fiber-optic components using supercontinuum radiation," Appl. Phys. B, vol. 81, no. 2, pp. 231-234, Jul. 2005, doi: 10.1007/s00340-005-1876-4.

[12] M. Kumar, M. N. Islam, F. L. Terry, C. C. Aleksoff, and D. Davidson, "High resolution line scan interferometer for solder ball inspection using a visible supercontinuum source," Opt. Express, OE, vol. 18, no. 21, pp. 22471-22484, Oct. 2010, doi: 10.1364/OE.18.022471.

[13] K. A. Serrels, M. K. Renner, and D. T. Reid, "Optical coherence tomography for non-destructive investigation of silicon integrated-circuits," Microelectronic Engineering, vol. 87, no. 9, pp. 1785-1791, Nov. 2010, doi: $10.1016 /$ j.mee.2009.10.011.

[14] M. Kumar et al., "Stand-off detection of solid targets with diffuse reflection spectroscopy using a high-power mid-infrared supercontinuum source," Appl. Opt., AO, vol. 51, no. 15, pp. 2794-2807, May 2012, doi: 10.1364/AO.51.002794.

[15] J. Kilgus, K. Duswald, G. Langer, and M. Brandstetter, "Mid-Infrared Standoff Spectroscopy Using a Supercontinuum Laser with Compact Fabry-Pérot Filter Spectrometers:," Applied Spectroscopy, Jan. 2018, doi: 10.1177/0003702817746696.

[16] X. Liu, S. Cheng, H. Liu, S. Hu, D. Zhang, and H. Ning, "A Survey on Gas Sensing Technology," Sensors (Basel), vol. 12, no. 7, pp. 9635-9665, Jul. 2012, doi: $10.3390 / \mathrm{s} 120709635$.

[17] J. Mellqvist and A. Rosén, "DOAS for flue gas monitoring-II. Deviations from the Beer-Lambert law for the U.V./visible absorption spectra of $\mathrm{NO}, \mathrm{NO} 2$, SO2 and NH3," Journal of Quantitative Spectroscopy and Radiative Transfer, vol. 56, no. 2, pp. 209-224, Aug. 1996, doi: 10.1016/0022-4073(96)00043-X.

[18] A. I. Adamu, F. E. Ozturk, and M. Bayindir, "Binary coded identification of industrial chemical vapors with an optofluidic nose," Appl. Opt., AO, vol. 55, no. 36, pp. 10247-10254, Dec. 2016, doi: 10.1364/AO.55.010247.

[19] J. M. Langridge, T. Laurila, R. S. Watt, R. L. Jones, C. F. Kaminski, and J. Hult, "Cavity enhanced absorption spectroscopy of multiple trace gas species using a supercontinuum radiation source," Opt. Express, OE, vol. 16, no. 14, pp. 10178-10188, Jul. 2008, doi: 10.1364/OE.16.010178.

[20] Y. Shao, J. Wang, H. Wu, J. Liu, I. A. Aksay, and Y. Lin, "Graphene Based Electrochemical Sensors and Biosensors: A Review," Electroanalysis, vol. 22, no. 10, pp. 1027-1036, 2010, doi: 10.1002/elan.200900571.

[21] P. K. Sekhar et al., "Application of commercial automotive sensor manufacturing methods for NOx/NH3 mixed potential sensors for on-board emissions control," Sensors and Actuators B: Chemical, vol. 144, no. 1, pp. 112-119, Jan. 2010, doi: 10.1016/j.snb.2009.10.045.
[22] N. Yamazoe and K. Shimanoe, "Theory of Power Laws for Semiconductor Gas Sensors," Sensors and Actuators B-chemical - SENSOR ACTUATOR B-CHEM, vol. 128, pp. 566-573, Jan. 2008, doi: 10.1016/j.snb.2007.07.036.

[23] B. Timmer, W. Olthuis, and A. van den Berg, "Ammonia sensors and their applications - a review," Sensors and Actuators B: Chemical, vol. 107, no. 2, pp. 666-677, Jun. 2005, doi: 10.1016/j.snb.2004.11.054.

[24] F. Berger, J.-B. Sanchez, and O. Heintz, "Detection of hydrogen fluoride using $\mathrm{SnO} 2$-based gas sensors: Understanding of the reactional mechanism," Sensors and Actuators B: Chemical, vol. 143, no. 1, pp. 152157, Dec. 2009, doi: 10.1016/j.snb.2009.08.054.

[25] V. Hernandez Bennetts, E. Schaffernicht, V. Pomareda, A. J. Lilienthal, S. Marco, and M. Trincavelli, "Combining Non Selective Gas Sensors on a Mobile Robot for Identification and Mapping of Multiple Chemical Compounds," Sensors, vol. 14, no. 9, pp. 17331-17352, Sep. 2014, doi: 10.3390/s140917331.

[26] R. B. Morrow and R. J. Wiler, "Ammonia Measurement in the IVC Microenvironment," J Am Assoc Lab Anim Sci, vol. 58, no. 2, pp. 184-189, Mar. 2019, doi: 10.30802/AALAS-JAALAS-18-000006.

[27] "MultiRAE IR MULTI-GAS MONITOR PGM-54. OPERATION AND MAINTENANCE MANUAL (Document No.: ) Rev.B - PDF Free Download." https://docplayer.net/21549638-Multirae-ir-multi-gasmonitor-pgm-54-operation-and-maintenance-manualdocument-no-008-4028-rev-b.html (accessed Apr. 28, 2020).

[28] Y. Lin, W. Jin, F. Yang, Y. Tan, and H. L. Ho, "Performance optimization of hollow-core fiber photothermal gas sensors," Opt. Lett., OL, vol. 42, no. 22, pp. 4712-4715, Nov. 2017, doi: 10.1364/OL.42.004712.

[29] P. Zhao et al., "Mode-phase-difference photothermal spectroscopy for gas detection with an anti-resonant hollow-core optical fiber," Nature Communications, vol. 11 , no. 1 , pp. $1-8$, Feb. 2020 , doi: 10.1038/s41467020-14707-0.

[30] J. J. Owen and W. L. Silver, "Greenhouse gas emissions from dairy manure management: a review of field-based studies," Global Change Biology, vol. 21, no. 2, pp. 550-565, 2015, doi: 10.1111/gcb.12687.

[31] S. G. Sommer and N. J. Hutchings, "Ammonia emission from field applied manure and its reduction-invited paper," European Journal of Agronomy, vol. 15, no. 1, pp. 1-15, Sep. 2001, doi: 10.1016/S11610301(01)00112-5.

[32] W. H. Schlesinger and A. E. Hartley, "A global budget for atmospheric NH3," Biogeochemistry, vol. 15, no. 3, pp. 191-211, Jan. 1992, doi: 10.1007/BF00002936.

[33] D. S. Chianese, C. A. Rotz, and T. L. Richard, "Simulation of Methane Emissions from Dairy Farms to Assess Greenhouse Gas Reduction Strategies," Transactions of the ASABE, vol. 52, no. 4, pp. 13131323, 2009, doi: 10.13031/2013.27781.

[34] S. C. Neubauer and J. P. Megonigal, "Moving Beyond Global Warming Potentials to Quantify the Climatic Role of Ecosystems," Ecosystems, vol. 18, no. 6, pp. 
1000-1013, Sep. 2015, doi: 10.1007/s10021-015-98794.

[35] J. M. Dudley, G. Genty, and S. Coen, "Supercontinuum generation in photonic crystal fiber," Rev. Mod. Phys., vol. 78, no. 4, pp. 1135-1184, Oct. 2006, doi: 10.1103/RevModPhys.78.1135.

[36] C. Hill, I. E. Gordon, R. V. Kochanov, L. Barrett, J. S. Wilzewski, and L. S. Rothman, "HITRANonline: An online interface and the flexible representation of spectroscopic data in the HITRAN database," Journal of Quantitative Spectroscopy and Radiative Transfer, vol. 177, pp. 4-14, Jul. 2016, doi: 10.1016/j.jqsrt.2015.12.012.

[37] L. Lundsbergnielsen, F. Hegelund, and F. M. Nicolaisen, "Analysis of the High-Resolution Spectrum of Ammonia (14NH3) in the Near-Infrared Region, 6400-6900 cm-1," Journal of Molecular Spectroscopy, vol. 162, no. 1, pp. 230-245, Nov. 1993, doi: 10.1006/jmsp.1993.1280.

[38] M. E. Webber, D. S. Baer, and R. K. Hanson, "Ammonia monitoring near $1.5 \mu \mathrm{m}$ with diode-laser absorption sensors," Appl. Opt., AO, vol. 40, no. 12, pp. 2031-2042, Apr. 2001, doi: 10.1364/AO.40.002031.

[39] J. Yang, B. Chen, J. Zhou, and Z. Lv, "A Low-Power and Portable Biomedical Device for Respiratory Monitoring with a Stable Power Source," Sensors (Basel, Switzerland), vol. 15, no. 8, p. 19618, Aug. 2015, doi: 10.3390/s150819618.

[40] Y. L. Hoo, S. Liu, H. L. Ho, and W. Jin, "Fast Response Microstructured Optical Fiber Methane Sensor With Multiple Side-Openings," IEEE Photonics Technology Letters, vol. 22, no. 5, pp. 296-298, Mar. 2010, doi: 10.1109/LPT.2009.2039016.

[41] J. Kilgus, P. Müller, P. M. Moselund, and M. Brandstetter, "Application of supercontinuum radiation for mid-infrared spectroscopy," in Optical Sensing and Detection IV, Apr. 2016, vol. 9899, p. 98990K, doi: 10.1117/12.2225886.

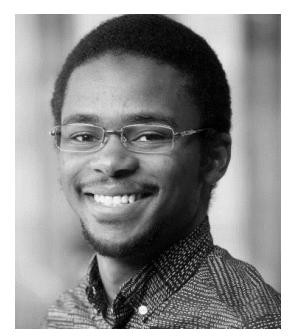

Abubakar I. Adamu is a Doctoral candidate in Department of Photonics Engineering at Technical University of Denmark. He received his B.Sc. in Engineering Physics from Gaziantep University. He was a visiting student at University of Chicago, USA in 2013/2014, where he studied on therapeutic modalities in Radiation Oncology, He has an M.Sc. in Material Science and Nanotechnology from Bilkent University, where he worked on artificial nose, for toxic gas sensing, at the National Nanotechnology research Center (UNAM), Turkey. He was a guest research student in University of Twente, Netherlands in 2015, where he worked on membranes for EUV lithography.

His Ph.D. focuses on Ultrafast nonlinear optics and Hollocore fibers for sensing and $\mathrm{SC}$ generation. He is a member of OSA, EPS, IEEE and SPIE, He was recently awarded the outstanding student paper award at SPIE Photonics West, 2019, San Francisco, USA.

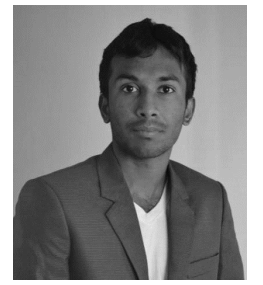

Manoj K. Dasa was born in Hyderabad, India in 1991. Mr. Dasa received his B. Tech. in Electronics and Communications Engineering from Jawaharlal Nehru Technological University, Hyderabad, India and M. Sc. in Electrical Communications Engineering with a specialization in Optoelectronics from University of Kassel, Germany in April 2016. In February 2017, he joined Technical University of Denmark as a Marie Curie Ph. D. fellow at the Department of Photonics Engineering. His Ph. D. focuses on developing high-pulse energy supercontinuum sources for multispectral photoacoustic applications in the extended near infrared region. Upon completion of his $\mathrm{PhD}$, he joined SHUTE Sensing Solutions as a Photonics Development Engineer.

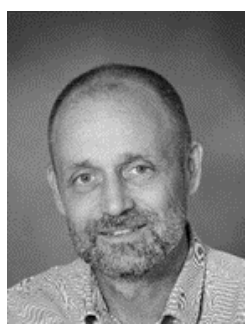

Ole Bang is a professor at the Technical University of Denmark and a Fellow of the OSA. He heads the Fiber Sensors \& Supercontinuum (FSS) Group, who develops photonic crystal fibers for sensing and supercontinuum lasers and use them for applications in imaging and spectroscopy. He has published 232 journal papers with 11083 ISI citations and has an ISI h-index of 57 . He holds several patents within supercontinuum lasers and polymer optical fiber sensors and is co-founder of SHUTE Sensing Solutions A/S and NORBLIS IVS.

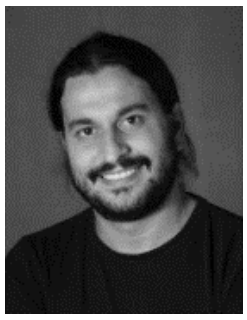

Christos Markos is an Associate Professor at the Technical University of Denmark. He leads the multi-material fiber fabrication and hollow-core gas sensing in the FSS group. He is an OSA Editor of Optical Material Express and member of SPIE, OSA and IEEE societies.

He has authored over 100 peer-reviewed journals and conference proceedings in fields of fiber optics, sensors and material science. His research interest includes ultrafast nonlinear optics, gas-filled hollow-core fibers, fabrication and design of specialty optical fibers for optogentics, neural interfaces and supercontinuum generation. He is a co-founder of NORBLIS IVS. 